\title{
Evaluation of Native Isolates of Bacillus thuringiensis against the Cotton Bollworm, Helicoverpa armigera (Hübner)
}

\author{
P. Swathi ${ }^{1}$, S. Ramesh Babu ${ }^{1}$, Devendra Jain ${ }^{2}$, M.K. Mahla ${ }^{1}$, \\ Deepika Kalyan ${ }^{1}$, P. Rokadia ${ }^{3}$ and Poonam Yadav ${ }^{4}$
}

\begin{abstract}
${ }^{1}$ Department of Entomology, ${ }^{2}$ Department of Molecular Biology and Biotechnology,
${ }^{4}$ Department of Plant Pathology, Rajasthan College of Agriculture, Maharana Pratap University of Agriculture and Technology (MPUAT), Udaipur-313001 (Rajasthan), India

${ }^{3}$ Agricultural Research Station, Maharana Pratap University of Agriculture and Technology (MPUAT), Banswara-327 001 (Rajasthan), India
\end{abstract}

*Corresponding author

\section{A B S T R A C T}

\section{Keywords}

Helicoverpa armigera, Bacillus thuringiensis, Larvae

Article Info

Accepted:

26 July 2020

Available Online:

10 August 2020
A total of twenty-eight native Bacillus thuringiensis strains were evaluated against neonate larvae of Helicoverpa armigera and found that three strains were effective with more than $80 \%$ cumulative mortality, followed by seven strains with $51-75$ and nine strains with more than $40 \%$ cumulative mortality at 7 days after treatment. Cumulative mortality of $83.3 \%$ was recorded in strain IBS1 which was statistically on par with each other and standard check Bt. kurstaki (HD-1) and also superior over other treatments. IBS5 and IBS14 also recorded higher cumulative mortality of neonate larvae of Helicoverpa armigera.

\section{Introduction}

Helicoverpa armigera (Hübner) (Lepidoptera; Noctuidae) is distributed from Europe, Africa, Asia and Australasia to the New World (Kriticos et al., 2015). In India, this pest is polyphagous in nature and present throughout the year and larval stage can survive and feed on 181 cultivated and wild plant species from about 45 families (Yogesh et al., 2016). Some of them are important agricultural crops like cotton, sorghum, groundnut, pigeonpea, chickpeas, tomato etc. Losses up to Rs.10, 000 million have been reported solely due to this pest in these crops (Indira, 2013 and Parmar et al., 2015; Chhangani, et al., 2018).

Insecticide resistance and resurgence in different crops led to the indiscriminate use of insecticides against $H$. armigera. This pest shows resistance to all the major insecticide classes and it has become increasingly 
difficult to control its population in India Indira, 2013. This has necessitated a search for more eco-friendly approaches to managing this pest (Reddy, et al., 2019). Integrated Pest Management (IPM) is one of the reliable methods to achieve stabilityin crop production, as it uses all the appropriate techniques in a way that is as compatible as possible to keep the pest population below the economic threshold (Mathur and Kishor, 1987). Biological control is a crucial tool in IPM strategies. Biocontrol agents viz., predators, parasitoids, virus and microbes, play a key role in natural pest management activities, due to their specificity in attack, effectiveness, and safety to non-target organisms.

Bacteria belonging to the Bacillaceae family have a wide range of insecticidal activity, and from which Bacillus thuringiensis (Bt) is the most commonly used and successfully employed microbial pesticide in agriculture. The B. thuringiensis, which accounted for $90 \%$ of the world market of biopesticides, is pathogenic to nearly 525 species of insectpests belonging to various orders (Khetan, 2001).

B. thuringiensis is a rod-shaped, grampositive, spore forming ubiquitous aerobic typical soil bacterium, first discovered by Ishiwata in Japan, 1901 and then by Berliner, 1911 in Germany (Baum et al., 1999). B. thuringiensis produces a parasporal crystal protein, known as "Cry" protein ( $\delta$ endotoxin), which is responsible for producing insect pest toxicity (Crickmore et al., 2014). Due to their varied toxicity levels, different kinds of isolates have been studied and used in agriculture. Many insect pests belonging to Lepidoptera can be managed effectively with Bt. Kurstaki (Nethravathi et al., 2010). Apart from major lepidopteran pests, $B t$ toxins are reported to show their toxicity on Diptera, Coleoptera, Orthoptera and also on nematodes, lice, mites, and aphids
(Rosas-Garcia, 2009). Many countries have concentrated their research in biological control to isolate new local strains to use in pest management effectively. Accordingly, the present investigation aimed to isolate local B. thuringiensis for the control of Helicoverpa armigera.

\section{Materials and Methods}

Bt like isolates based on crystal morphology and twenty-eight $\mathrm{Bt}$ isolates were purified as single colony and maintained on specific medium (LB and T3 medium) and stored in glycerol stocks at -70_C for future studies.

\section{Preparation of spore crystal mixture (Lenin et al., 2001)}

The $B t$ culture was maintained in T3 agar plates at $4^{\circ} \mathrm{C}$. Single colony of $\mathrm{Bt}$ was inoculated into $4 \mathrm{ml} \mathrm{T3}$ broth and kept in a shaker, at $30^{\circ} \mathrm{C}$ temperature at $200 \mathrm{rpm}$ for $48 \mathrm{~h}$. After overnight growth, the inoculum was added to $250 \mathrm{ml}$ flask containing $25 \mathrm{ml}$ $\mathrm{T} 3$ medium and incubated at $30^{\circ} \mathrm{C}$ on a rotary shaker maintained at $200 \mathrm{rpm}$ for nearly $48 \mathrm{~h}$. When more than 90 per cent lysis was reached, the culture was transferred to $4^{\circ} \mathrm{C}$, at least half-an-hour before harvesting. The T3 broth containing spore-crystal mixture was centrifuged at $10,000 \mathrm{Xg}$ at $4{ }^{\circ} \mathrm{C}$. The pellet obtained was resuspended in ice cold $1 \mathrm{ml}$ Tris-EDTA buffer [Tris $10 \mathrm{mM}$, EDTA $1 \mathrm{mM}$, $\mathrm{pH} 8.0$ with $1 \mathrm{mM}$ phenylmethyl sulphonyl fluoride (PMSF)] and washed once with $1 \mathrm{ml}$ ice-cold $0.5 \mathrm{M} \mathrm{NaCl}$ at $10,000 \mathrm{X} \mathrm{g}$ for 10 min followed by two washes with $1 \mathrm{ml}$ TrisEDTA buffer at the same speed and time. Finally, the spore-crystal pellet was resuspended in $100 \mu \mathrm{l}$ sterile distilled water with $1 \mathrm{mM}$ PMSF and stored in $-20^{\circ} \mathrm{C}$.

Bioassay of local Bacillus thuringiensis isolates against Helicoverpa armigera

Mass screening of $B$. thuringiensis isolates 
including commercial $\mathrm{Bt}$ (B. thurigiensis kurstakiHD-1) for toxicity against $H$. armigera was performed using relatively higher concentration $\left(500 \mu \mathrm{g} \mathrm{ml}^{-1}\right)$ of sporecrystal mixtures. First instar larvae were used for bioassay by diet incorporation method (Song et al., 2003 and Lone et al., 2017). Thirty larvae per treatment were used. In the control, the culture was substituted with sterile distilled water. Mortality was recorded after 24 hrs to 7 days; larvae were scored as dead if they failed to respond to gentle probing. The experiment was set in triplicate, making total number of larvae tested per treatment to 72 . The mortality observed was corrected to control mortality by Abbott's formula (Abbott, 1925). The data were subjected to ANOVA one way using minitab 19 online statistical package.

\section{Results and Discussion}

Inhibition of larval growth and toxic effects of twenty eight native isolates of $B$. thuringiensis against Helicoverpa armigera neonates after 1 day of treatment till 7 days was recorded. The reference strain exhibited the highest mortality rate of $9.72,26.39,26.38$ and 23.61 at $1,3,5$ and 7 days after the treatment. Among all the isolates IBS 1 showed higher mortality rates of 9.72, 22.22, 30.55, 20.88 after 1,3, 5 and 7 days of exposure, respectively with a cumulative of 83.38 which was comparable with the standard strain. It was followed by IBS 14 with a similar mortality rate of 81.94. Isolates IBS 17, IBS28, IBS 42, and IBS 41 showed cumulative mortality rate of $64.15,61.11$ and 61.0, respectively. Isolates ISB32, ISB36, ISB19, ISB16 also showed promising toxic effects against Helicoverpa larvae with mortality rates of 56.93, 59.71, 50.38 and 54.15 , respectively. They are followed by IBS3, IBS8, IBS9, IBS10, IBS18, IBS12 and IBS21 isolates with $16.66,16.66,19.44$, $20.83,16.88,13.88$ and 18.05 after 7 days of treatment (Table 1).

Table.1 Bioassay of native Bt isolates against neonate larvae of $H$. armigera

\begin{tabular}{|c|c|c|c|c|c|c|}
\hline S. No. & Strain/Isolate & $1^{\text {st }}$ day & $3^{\text {rd }}$ day & $5^{\text {th }}$ day & 7th day & Cumulative \\
\hline 1 & IBS1 & $\begin{array}{c}9.72 \\
(18.06) \mathrm{a}-\mathrm{e}^{*}\end{array}$ & $\begin{array}{c}22.22 \\
(28.08) \mathrm{ab}\end{array}$ & $\begin{array}{c}30.55 \\
(33.56) \mathrm{a}\end{array}$ & $\begin{array}{c}20.88 \\
(27.17) \mathrm{bcd}\end{array}$ & $\begin{array}{c}83.38 \\
(65.98) \mathrm{ab}\end{array}$ \\
\hline 2 & IBS2 & $\begin{array}{c}1.39 \\
(3.94) \text { ef }\end{array}$ & $\begin{array}{c}4.16 \\
(9.473) \mathrm{ij}\end{array}$ & $\begin{array}{c}8.66 \\
(17.08) \mathrm{k}-\mathrm{n}\end{array}$ & $\begin{array}{c}15.27 \\
\text { (22.92)ef }\end{array}$ & $\begin{array}{c}29.49 \\
(32.85) \mathrm{m}\end{array}$ \\
\hline 3 & IBS3 & $\begin{array}{l}2.77 \\
(7.89) \text { def }\end{array}$ & $\begin{array}{c}5.55 \\
(13.36) \text { hij }\end{array}$ & $\begin{array}{c}15.27 \\
(22.96) \mathrm{fgh}\end{array}$ & $\begin{array}{c}16.66 \\
(24.04) \text { def }\end{array}$ & $\begin{array}{c}40.26 \\
(39.37) \mathrm{i}-\mathrm{m}\end{array}$ \\
\hline 4 & IBS5 & $\begin{array}{c}15.27 \\
(22.69) \mathrm{a}\end{array}$ & $\begin{array}{c}27.77 \\
(31.80) \mathrm{a}\end{array}$ & $\begin{array}{c}19.44 \\
(26.13) \mathrm{de}\end{array}$ & $\begin{array}{c}18.05 \\
(24.98) \mathrm{cdef}\end{array}$ & $\begin{array}{c}80.54 c \\
(63.98) a b c\end{array}$ \\
\hline 5 & IBS6 & $\begin{array}{c}5.55 \\
(11.16) b-f\end{array}$ & $\begin{array}{c}9.72 \\
(18.060) \text { e-j }\end{array}$ & $\begin{array}{c}15.40 \\
(23.06) f g h\end{array}$ & $\begin{array}{c}8.33 \\
(16.74) \mathrm{g}\end{array}$ & $\begin{array}{c}39.01 \\
(38.58) \mathrm{i}-\mathrm{m}\end{array}$ \\
\hline 6 & IBS8 & $\begin{array}{c}4.16 \\
(11.83) \mathrm{c}-\mathrm{f}\end{array}$ & $\begin{array}{c}8.66 \\
(17.08) \mathrm{f}-\mathrm{j}\end{array}$ & $\begin{array}{c}12.50 \\
(20.70) \mathrm{h}-\mathrm{k}\end{array}$ & $\begin{array}{c}16.66 \\
(23.95) \mathrm{def}\end{array}$ & $\begin{array}{c}41.99 \\
(40.36) \mathrm{h}-\mathrm{m}\end{array}$ \\
\hline 7 & IBS9 & $\begin{array}{c}6.94 \\
(15.10) \mathrm{a}-\mathrm{f}\end{array}$ & $\begin{array}{c}9.72 \\
(18.06) \text { e-j }\end{array}$ & $\begin{array}{c}12.77 \\
(20.93) g-j\end{array}$ & $\begin{array}{c}19.44 \\
(26.10) \text { bcde }\end{array}$ & $\begin{array}{c}48.88 \\
(44.35) \mathrm{e}-1\end{array}$ \\
\hline 8 & IBS10 & $\begin{array}{c}6.94 \\
(15.10) \mathrm{a}-\mathrm{f}\end{array}$ & $\begin{array}{c}8.33 \\
(16.74) \mathrm{f}-\mathrm{j}\end{array}$ & $\begin{array}{c}9.72 \\
(15.14) \mathrm{j}-\mathrm{m}\end{array}$ & $\begin{array}{c}20.83 \\
(27.05) \mathrm{bcd}\end{array}$ & $\begin{array}{c}45.82 \\
(42.58) \mathrm{f}-\mathrm{m}\end{array}$ \\
\hline 9 & IBS 12 & $\begin{array}{c}5.55 \\
(13.47) b-f\end{array}$ & $\begin{array}{c}9.72 \\
(18.06) e-j\end{array}$ & $\begin{array}{c}11.11 \\
(19.45) \mathrm{i}-1\end{array}$ & $\begin{array}{c}13.88 \\
(21.81) f\end{array}$ & $\begin{array}{c}40.26 \\
(39.36) \mathrm{i}-\mathrm{m}\end{array}$ \\
\hline
\end{tabular}




\begin{tabular}{|c|c|c|c|c|c|c|}
\hline 10 & IBS 14 & $\begin{array}{c}11.11 \\
(19.38) \mathrm{a}-\mathrm{d}\end{array}$ & $\begin{array}{c}26.39 \\
(30.90) \mathrm{a}\end{array}$ & $\begin{array}{c}20.83 \\
(27.15) \mathrm{cd}\end{array}$ & $\begin{array}{c}23.61 \\
(29.04) a b\end{array}$ & $\begin{array}{c}81.94 \\
(64.95) \mathrm{ab}\end{array}$ \\
\hline 11 & IBS 16 & $\begin{array}{c}4.16 \\
(11.83) c-f\end{array}$ & $\begin{array}{c}15.27 \\
(22.92) b-f\end{array}$ & $\begin{array}{c}12.50 \\
(20.64) h-k\end{array}$ & $\begin{array}{c}21.89 \\
(28.01) \text { bcde }\end{array}$ & $\begin{array}{c}54.15 \\
(47.36) \mathrm{e}-\mathrm{i}\end{array}$ \\
\hline 12 & IBS17 & $\begin{array}{c}12.50 \\
(20.49) a b c\end{array}$ & $\begin{array}{c}18.05 \\
(25.070) \text { bcd }\end{array}$ & $\begin{array}{c}13.88 \\
(21.86) g-i\end{array}$ & $\begin{array}{c}26.39 \\
(30.79) \mathrm{a}\end{array}$ & $\begin{array}{c}70.82 \\
(57.47) \text { bcd }\end{array}$ \\
\hline 13 & IBS 18 & $\begin{array}{c}4.160 \\
(11.83) \mathrm{c}-\mathrm{f}\end{array}$ & $\begin{array}{c}16.66 \\
(24.040) b-e\end{array}$ & $\begin{array}{c}9.72 \\
(18.14) \mathrm{j}-\mathrm{m}\end{array}$ & $\begin{array}{c}16.88 \\
\text { (24.22)def }\end{array}$ & $\begin{array}{c}47.42 \\
(43.53) \mathrm{f}-1\end{array}$ \\
\hline 14 & IBS19 & $\begin{array}{c}11.11 \\
(19.38) \mathrm{a}-\mathrm{d}\end{array}$ & $\begin{array}{c}8.55 \\
(16.980) \mathrm{f}-\mathrm{j}\end{array}$ & $\begin{array}{c}16.66 \\
\text { (24.04)efg }\end{array}$ & $\begin{array}{c}14.05 \\
(21.95) f\end{array}$ & $\begin{array}{c}50.38 \\
(45.21) e-j\end{array}$ \\
\hline 15 & IBS20 & $\begin{array}{l}4.16 \\
(9.52) \mathrm{c}-\mathrm{f}\end{array}$ & $\begin{array}{c}9.72 \\
(18.06) \text { e-j }\end{array}$ & $\begin{array}{c}6.27 \\
(14.42) \mathrm{mn}\end{array}$ & $\begin{array}{c}13.88 \\
(21.81) f\end{array}$ & $\begin{array}{c}34.04 \\
(35.62) \mathrm{j}-\mathrm{m}\end{array}$ \\
\hline 16 & IBS21 & $\begin{array}{c}6.94 \\
(12.48) a-f\end{array}$ & $\begin{array}{c}8.22 \\
(16.637) \mathrm{f}-\mathrm{j}\end{array}$ & $\begin{array}{c}11.11 \\
(19.44) \mathrm{i}-1\end{array}$ & $\begin{array}{c}18.05 \\
(24.91) \mathrm{cdef}\end{array}$ & $\begin{array}{c}44.33 \\
(41.690) \mathrm{g}-\mathrm{m}\end{array}$ \\
\hline 17 & IBS22 & $\begin{array}{c}12.50 \\
(20.70) \mathrm{abc}\end{array}$ & $\begin{array}{c}9.72 \\
(18.06) \text { e-j }\end{array}$ & $\begin{array}{c}13.89 \\
(21.81) \mathrm{ghi}\end{array}$ & $\begin{array}{c}15.27 \\
\text { (22.63)ef }\end{array}$ & $\begin{array}{c}51.38 \\
(45.79) \mathrm{e}-\mathrm{i}\end{array}$ \\
\hline 18 & IBS23 & $\begin{array}{l}2.77 \\
\text { (7.89) def }\end{array}$ & $\begin{array}{c}6.93 \\
(15.05) \text { ghij }\end{array}$ & $\begin{array}{c}6.94 \\
(15.22) \mathrm{mn}\end{array}$ & $\begin{array}{c}14.05 \\
(21.81) f\end{array}$ & $\begin{array}{c}30.53 \\
(33.47) \mathrm{m}\end{array}$ \\
\hline 19 & IBS24 & $\begin{array}{c}4.16 \\
(11.83) \mathrm{c}-\mathrm{f}\end{array}$ & $\begin{array}{c}7.01 \\
(15.12) g-j\end{array}$ & $\begin{array}{c}8.33 \\
(16.74) \mathrm{k}-\mathrm{n}\end{array}$ & $\begin{array}{c}15.27 \\
\text { (22.92)ef }\end{array}$ & $\begin{array}{c}34.78 \\
(36.07) \mathrm{j}-\mathrm{m}\end{array}$ \\
\hline 20 & IBS26 & $\begin{array}{c}4.16 \\
(9.52) \mathrm{c}-\mathrm{f}\end{array}$ & $\begin{array}{c}9.72 \\
(18.06) \text { e-j }\end{array}$ & $\begin{array}{c}5.55 \\
(13.54) \mathrm{mn}\end{array}$ & $\begin{array}{c}13.88 \\
(21.81) \mathrm{f}\end{array}$ & $\begin{array}{c}33.32 \\
(35.22) 1 \mathrm{~m}\end{array}$ \\
\hline 21 & IBS40 & $\begin{array}{c}0.00 \\
(0.00) \mathrm{f}\end{array}$ & $\begin{array}{c}9.94 \\
(18.30) \text { e-j }\end{array}$ & $\begin{array}{c}7.05 \\
(15.17) \mathrm{mn}\end{array}$ & $\begin{array}{c}16.66 \\
\text { (23.95)def }\end{array}$ & $\begin{array}{c}33.66 \\
(35.40) \mathrm{klm}\end{array}$ \\
\hline 22 & IBS44 & $\begin{array}{c}5.55 \\
(13.47) b-f\end{array}$ & $\begin{array}{c}13.89 \\
(21.81) \mathrm{c}-\mathrm{g}\end{array}$ & $\begin{array}{c}10.05 \\
(18.42) \mathrm{i}-\mathrm{m}\end{array}$ & $\begin{array}{c}15.27 \\
(22.92) \mathrm{ef}\end{array}$ & $\begin{array}{c}44.76 \\
\text { (41.98)f-m }\end{array}$ \\
\hline 23 & IBS42 & $\begin{array}{c}9.72 \\
(20.49) \text { a-e }\end{array}$ & $\begin{array}{c}12.50 \\
(20.49) d-h\end{array}$ & $\begin{array}{c}15.29 \\
(22.96) f g h\end{array}$ & $\begin{array}{c}23.61 \\
(28.89) a b\end{array}$ & $\begin{array}{c}61.11 \\
\text { (51.41)def }\end{array}$ \\
\hline 24 & IBS41 & $\begin{array}{c}13.89 \\
(21.81) \mathrm{ab}\end{array}$ & $\begin{array}{c}16.66 \\
(23.95) b-e\end{array}$ & $\begin{array}{c}16.66 \\
\text { (24.04)efg }\end{array}$ & $\begin{array}{c}13.88 \\
(21.81) f\end{array}$ & $\begin{array}{c}61.10 \\
\text { (51.47)def }\end{array}$ \\
\hline 25 & IBS28 & $\begin{array}{c}8.33 \\
(16.42) a-f\end{array}$ & $\begin{array}{c}20.83 \\
(27.05) \mathrm{abc}\end{array}$ & $\begin{array}{c}18.05 \\
(25.10) \mathrm{de}\end{array}$ & $\begin{array}{c}16.94 \\
\text { (24.27)def }\end{array}$ & $\begin{array}{c}64.15 \\
(53.23) \mathrm{cde}\end{array}$ \\
\hline 26 & IBS30 & $\begin{array}{c}4.16 \\
(9.52) \mathrm{c}-\mathrm{f}\end{array}$ & $\begin{array}{c}12.50 \\
(20.70) \text { d-h }\end{array}$ & $\begin{array}{c}19.44 \\
(26.12) \mathrm{de}\end{array}$ & $\begin{array}{c}14.05 \\
(21.81) \mathrm{f}\end{array}$ & $\begin{array}{c}49.99 \\
(44.97) \mathrm{e}-\mathrm{k}\end{array}$ \\
\hline 27 & IBS32 & $\begin{array}{c}6.94 \\
(15.10) a-f\end{array}$ & $\begin{array}{c}11.11 \\
(19.38) \mathrm{d}-\mathrm{i}\end{array}$ & $\begin{array}{c}23.61 \\
(29.06) \mathrm{bc}\end{array}$ & $\begin{array}{c}15.27 \\
(22.92) \mathrm{ef}\end{array}$ & $\begin{array}{c}56.93 \\
(48.97) d-h\end{array}$ \\
\hline 28 & IBS36 & $\begin{array}{c}4.16 \\
(9.52) \mathrm{c}-\mathrm{f}\end{array}$ & $\begin{array}{c}13.89 \\
(21.81) \mathrm{c}-\mathrm{g}\end{array}$ & $\begin{array}{c}24.99 \\
(29.98) b\end{array}$ & $\begin{array}{c}16.66 \\
\text { (24.04)def }\end{array}$ & $\begin{array}{c}59.71 \\
(50.59) d-g\end{array}$ \\
\hline 29 & Bt kurstaki & $\begin{array}{c}9.72 \\
(18.06) \mathrm{a}-\mathrm{e}\end{array}$ & $\begin{array}{c}26.39 \\
(30.90) a\end{array}$ & $\begin{array}{c}26.38 \\
(30.87) b\end{array}$ & $\begin{array}{c}23.61 \\
(29.92) a b\end{array}$ & $\begin{array}{c}87.49 \\
(69.31)\end{array}$ \\
\hline a30 & Control & $\begin{array}{r}0.00 \\
(0.00) \mathrm{f}\end{array}$ & $\begin{array}{c}2.94 \\
(9.75) \mathrm{j}\end{array}$ & $\begin{array}{c}1.39 \\
\text { (3.89)o }\end{array}$ & $\begin{array}{c}1.99 \\
(8.04) \mathrm{h}\end{array}$ & $\begin{array}{c}6.32 \\
(14.17) n\end{array}$ \\
\hline
\end{tabular}

Figures in parentheses are arcsine transformed values; * Values followed by different letters (a, $\mathrm{b}, \mathrm{c}, \mathrm{e}, \mathrm{f}, \mathrm{g}, \mathrm{h}, \mathrm{i}, \mathrm{j})$ were significantly different $(\mathrm{P}<0.05$, Tukey post-test $)$ 
Figure.1 Insecticidal Activity of Different Native Bt isolates against the First Instar Larvae of Helicoverpa armigera. Cumulative mortality was measured at the 7th day of assay. Mortality differed between different Bt isolates significantly and measured at $\mathrm{P}<0.05$, Tukey test

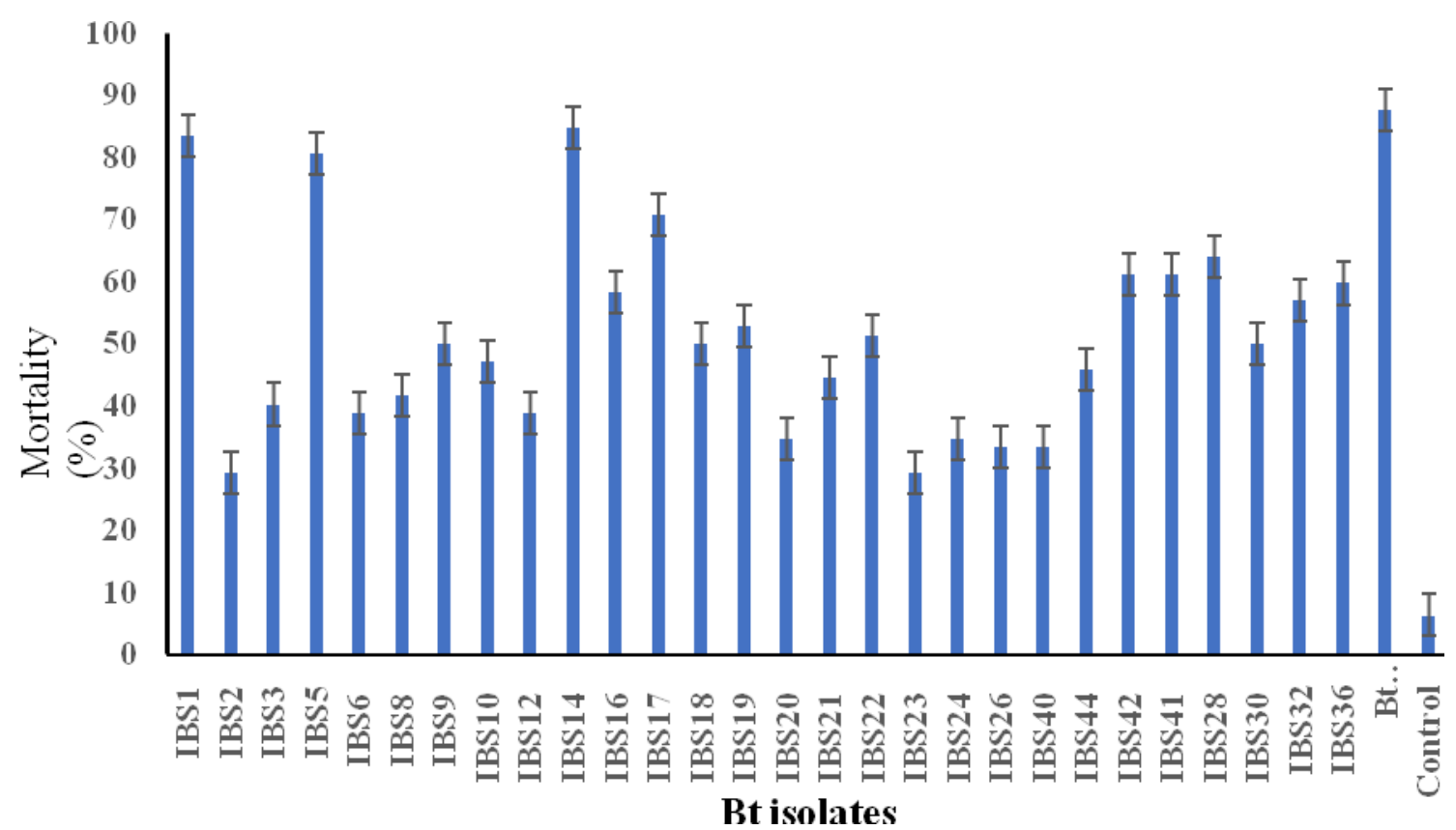

Among the tenBt isolates, two were effective against Spodoptera littoralis and two more $\mathrm{Bt}$ isolates toxicity against Helicoverpa armigera (Merdan et al., 2010). Similarly, Patel et al., (2018) isolated native Bt isolates and sporecrystal mixture of $B$. thuringiensis was tested at $10^{7}, 10^{8}$ and $10^{9}$ concentrations with that of Btk HD-73 (standard check). Significant differences in all the screened Bt isolates were observed on the per cent mortality of $H$. armigera.

\section{Acknowledgment}

The authors are highly thankful to Zonal Director Research, ARS, Banswara for providing necessary lab facilities for conducting the experiment. We also acknowledge the Department of Molecular Biology and Biotechnology, RCA, MPUAT,
Udaipur and thanks to Rashtriya Krishi Vikas Yojana (RKVY), GoI for supporting this work for using facilities created under this project.

\section{References}

Abbott, W.S. (1925). A method of computing the effectiveness of an insecticide. Journal of Economic Entomology, 18: 265-267.

Ankit, S. Patel., Harsha N., Shelat. and Hiren, K. Patel. (2018). Isolation and Insecticidal Potential of Native Bacillus thuringiensis against Helicoverpa armigera and Spodoptera litura. Int.J.Curr.Microbiol.App.Sci, $7(2)$, 1330-1339.

Ashokan, R. and Puttaswamy. (2007) Isolation and Characterization of 
Bacillus thuringiensis Berliner from soil, leaf, seed dust and insect cadavers. Journal of Biological Control, 21, 8390.

Baum, J.A., Johnson, T.B., Carlton, B.C. (1999).Bacillus thuringiensis: natural and recombinant bio-insecticide products. Biopesticides: Use and Delivery. Humana Press, Totowa, pp 189-210.

Crickmore, N., Zeigler, D.R., Feitelson, J., Schnepf, E., Van Rie, J., Lereclus, D., Baum, J. and Dean, D.H. (2014).Bacillus thuringiensis Toxin Nomenclature.

Chhangani, . G., A. Vyas, M.K.Mahla, A. Mordia, M.K.Tali and B. Singh (2018). Effect of abiotic factors on seasonal incidence of gram Pod borer, Helicoverpa armigera (Hubner) in gram. Indian J. Appl. Ent. 32(1):1-3.

Ibarra, J.E., del Rincon, M.C. Orduz, S., Noriega, D., Benintende, G., Monnerat, R., Regis, L., de Oliveira, C.M., Lanz, H., Rodriguez, M.H., Sanchez, J., Pena, G. and Bravo. A. (2003). Diversity of Bacillus thuringiensis strains from Latin America with insecticidal activity against different mosquito species. Applied and Environmental Microbiology, 69, 5269-5274.

Indira C. A. (2013).Survey of insecticide resistance in Helicoverpa armigera in central and South Indian cotton ecosystems. International Journal of Research in BioScience,. 2(1), 37-43.

Kalha, C.S., Singh, R.P., Kang, S.S., Hunjan, M.S., Gupta, V., Sharma, Rishu. (2014). Entomopathogenic viruses and bacteria for insect-pest control. In: Abrol DP (ed) Integrated pest management: current concepts and ecological perspectives. Academic, San Diego, pp 225-244.

Khetan, S.K. (2001). Microbial Pest Control, pp: 199-210. Marcel Dekker, New
York, USA.

Ishiwata, S. (1901). On a kind of severe flacherie (sotto disease). Dainihon Sanshi Kaiho, 114:1-5.

Konecka, Edyta; Baranek, Jakub; Hrycak, Anita \& Kaznowski, Adam. (2012). Insecticidal Activity of Bacillus thuringiensis Strains Isolated from Soil and Water. The Scientific World Journal. 2012. 710501.

Kriticos, DJ., Ota N., Hutchison WD., Beddow J., Walsh T., Tay WT. (2015). The Potential Distribution of Invading Helicoverpa armigera in North America: Is It Just a Matter of Time? PLoS one. 10(3): $\mathrm{e} 0119618$. doi:10.1371/journal.pone.0119618.

Lone, Song, F., Abdul Malik, Jasdeep, C. and Padaria. (2017) Selection and characterization of Bacillus thuringiensis strains from northwestern Himalayas toxic against Helicoverpa armigera. Microbiology, 6, 484.

Mathur, K.C., Reddy, P.R., Rajamali, S. and Moorthy, B. T. S. (1999) Integrated pest management of rice to improve productivity and sustainability. Oryza, 36(3,: 195-207.

Meihiar, M., Ahmad, M., Al-Zyoud, F. and Amer, K. (2015). Environmental Distribution, Frequency and Toxicity of Bacillus thuringiensis in Syria. Annual Research and Review in Biology, 5, 174-183.

Merdan, A., Salama, H.S., Labib, E., Ragaei and Mand, A.E.G. (2010). Bacillus thuringiensis isolates from soil and diseased insects in Egyptian cotton fields and their activity against lepidopteran insects. Archives of Phytopathology and Plant Protection, 43 (12), 1165-1176.

Morales-Ramos, J.A., Guadalupe Rojas, M. and Shapro-Ilan, D.L. (Eds.) (2014) Mass Production of Beneficial Organisms. Elsevier, Amsterdam, pp. 
483-517.

Nethravathi, C. J., Hugar, P. S., Krishnaraj, P. U., Vastrad, A. S., Awaknavar, J. S. (2010). Bio-efficacy of native Sikkim Bacillus thuringiensis (Berliner) isolates against lepidopteran insects. Journal of Biopesticides, 3, 448-451.

Parmar K, Thakur K, Marabi RS. (2015). Effect of sowing dates and weather parameters on the incidence of Helicoverpa armigera (Hubner) in chickpeas. The Bioscan. 10(1), 93-96.

Rampersad, J. and David, A. (2005) A Bacillus thuringiensis isolation method utilizing a novel strain, low selection and high throughput produced atypical results. Bio Medical Central microbiology, 5: 1-11.

Reddy, K.V.N., Vyas, A., Lekha and Chhangani, G. (2019). Bioefficacy of insecticides against fruit borer, Helicoverpa armigera (Hub.) infesting tomato. Indian J. Appl. Ent. 33(1):2428 .

Rosas-Garcia N.M. (2009). Biopesticide production from Bacillus thuringiensis: an environmentally friendly alternative. Recent Patents on Biotechnology, 3, 28-36

Song, F., Zhang, J., Gu, A., Wu, Y., Han, L., He, K., Chen, Z., Yao, J., Hu, Y., Li, G. (2003). Identification of cry1 type genes of Bacillus thuringiensis and characterization of a novel cryltype gene. Applied Environmental Microbiology, 69, 5207-5211.

Unalmis, S., Ayvaz, A., Yilmaz, S., Azizoglu, U. (2015) Molecular screening and bioactivity of native Bacillus thuringiensis isolates. Journal of Agriculture science and Technolog, 17, 1197-1207.

Yogesh, Rajnish Kumar, Gyan Prakash Morya. (2016). A Study on Larval Population and Pod Damage due to Helicoverpa armigera in Pigeon pea under Eastern Uttar Pradesh Conditions. International Journal of Theoretical \& Applied Sciences, 8(2), 29-32.

\section{How to cite this article:}

Swathi, P., S. Ramesh Babu, Devendra Jain, M.K. Mahla, Deepika Kalyan, P. Rokadia and Poonam Yadav. 2020. Evaluation of Native Isolates of Bacillus thuringiensis against the Cotton Bollworm, Helicoverpa armigera (Hübner). Int.J.Curr.Microbiol.App.Sci. 9(08): 34503456. doi: https://doi.org/10.20546/ijcmas.2020.908.399 